NASA/TM-2007-214838

ARL-TR-3930
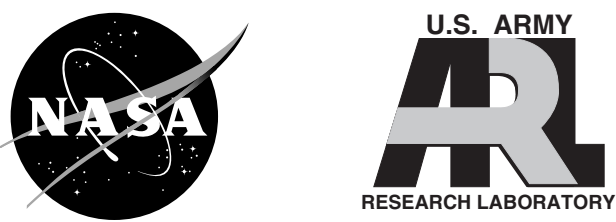

\title{
Towards Autonomous Inspection of Space Systems Using Mobile Robotic Sensor Platforms
}

Edmond Wong

Glenn Research Center, Cleveland, Ohio

Ashraf Saad

Armstrong Atlantic State University, Savannah, Georgia

Jonathan S. Litt

U.S. Army Research Laboratory, Glenn Research Center, Cleveland, Ohio 


\section{NASA STI Program . . . in Profile}

Since its founding, NASA has been dedicated to the advancement of aeronautics and space science. The NASA Scientific and Technical Information (STI) program plays a key part in helping NASA maintain this important role.

The NASA STI Program operates under the auspices of the Agency Chief Information Officer. It collects, organizes, provides for archiving, and disseminates NASA's STI. The NASA STI program provides access to the NASA Aeronautics and Space Database and its public interface, the NASA Technical Reports Server, thus providing one of the largest collections of aeronautical and space science STI in the world. Results are published in both non-NASA channels and by NASA in the NASA STI Report Series, which includes the following report types:

- TECHNICAL PUBLICATION. Reports of completed research or a major significant phase of research that present the results of NASA programs and include extensive data or theoretical analysis. Includes compilations of significant scientific and technical data and information deemed to be of continuing reference value. NASA counterpart of peer-reviewed formal professional papers but has less stringent limitations on manuscript length and extent of graphic presentations.

- TECHNICAL MEMORANDUM. Scientific and technical findings that are preliminary or of specialized interest, e.g., quick release reports, working papers, and bibliographies that contain minimal annotation. Does not contain extensive analysis.

- CONTRACTOR REPORT. Scientific and technical findings by NASA-sponsored contractors and grantees.
- CONFERENCE PUBLICATION. Collected papers from scientific and technical conferences, symposia, seminars, or other meetings sponsored or cosponsored by NASA.

- SPECIAL PUBLICATION. Scientific, technical, or historical information from NASA programs, projects, and missions, often concerned with subjects having substantial public interest.

- TECHNICAL TRANSLATION. Englishlanguage translations of foreign scientific and technical material pertinent to NASA's mission.

Specialized services also include creating custom thesauri, building customized databases, organizing and publishing research results.

For more information about the NASA STI program, see the following:

- Access the NASA STI program home page at http://www.sti.nasa.gov

- E-mail your question via the Internet to help@sti.nasa.gov

- Fax your question to the NASA STI Help Desk at 301-621-0134

- Telephone the NASA STI Help Desk at 301-621-0390

- Write to: NASA Center for AeroSpace Information (CASI) 7115 Standard Drive Hanover, MD 21076-1320 
NASA/TM-2007-214838

ARL-TR-3930
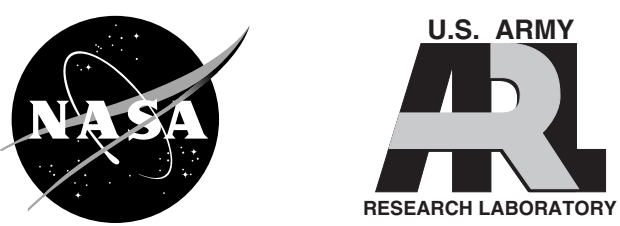

AIAA-2006-7411

\section{Towards Autonomous Inspection of Space Systems Using Mobile Robotic Sensor Platforms}

Edmond Wong

Glenn Research Center, Cleveland, Ohio

Ashraf Saad

Armstrong Atlantic State University, Savannah, Georgia

Jonathan S. Litt

U.S. Army Research Laboratory, Glenn Research Center, Cleveland, Ohio

Prepared for the

Space 2006

sponsored by the American Institute of Aeronautics and Astronautics

San Jose, California, September 19-21, 2006

National Aeronautics and

Space Administration

Glenn Research Center

Cleveland, Ohio 44135 


\section{Acknowledgments}

The authors would like to acknowledge the significant contributions to this research by the following: Lawrence C. Greer and Michael J. Krasowski of NASA Glenn Research Center; and Joseph Auchter from Florida State University.

Trade names and trademarks are used in this report for identification only. Their usage does not constitute an official endorsement, either expressed or implied, by the National Aeronautics and Space Administration.

Level of Review: This material has been technically reviewed by technical management.

Available from

NASA Center for Aerospace Information 7115 Standard Drive

Hanover, MD 21076-1320
National Technical Information Service 5285 Port Royal Road Springfield, VA 22161 


\title{
Towards Autonomous Inspection of Space Systems Using Mobile Robotic Sensor Platforms
}

\author{
Edmond Wong \\ National Aeronautics and Space Administration \\ Glenn Research Center \\ Cleveland, Ohio 44135 \\ Ashraf Saad \\ Armstrong Atlantic State University \\ Savannah, Georgia 31419 \\ Jonathan S. Litt \\ U.S. Army Research Laboratory \\ Glenn Research Center \\ Cleveland, Ohio 44135
}

\begin{abstract}
The space transportation systems required to support NASA's Exploration Initiative will demand a high degree of reliability to ensure mission success. This reliability can be realized through autonomous fault/damage detection and repair capabilities. It is crucial that such capabilities are incorporated into these systems since it will be impractical to rely upon Extra-Vehicular Activity (EVA), visual inspection or tele-operation due to the costly, laborintensive and time-consuming nature of these methods. One approach to achieving this capability is through the use of an autonomous inspection system comprised of miniature mobile sensor platforms that will cooperatively perform high-confidence inspection of space vehicles and habitats. This paper will discuss the efforts to develop a small scale demonstration test-bed to investigate the feasibility of using autonomous mobile sensor platforms to perform inspection operations. Progress will be discussed in technology areas including: the hardware implementation and demonstration of robotic sensor platforms, the implementation of a hardware test-bed facility, and the investigation of collaborative control algorithms.
\end{abstract}

\section{Introduction}

As space exploration systems become more complex and operate over longer periods of time in harsh environments, it will become impractical to depend on human intervention to maintain day to day safe and reliable operation. Currently, inspection and repair of existing human-occupied space transportation systems and platforms is heavily labor intensive, costly and time consuming. Examples in current systems that required extensive human intervention include the recent air leak in the International Space Station (which required the crew to devote all their effort over an extended period of time to locate and repair the leak), and the manual process involved in shuttle heattile inspection and repair. As a result, there will be a need for the development of autonomous solutions capable of high-confidence inspection and repair in order for future space systems to operate successfully.

To fulfill this need, this paper proposes a mobile sensor-based inspection system that emphasizes the use of simple, low-cost robotic sensor platforms that can be dispatched to traverse the structures and component surfaces of future space systems. The goal to comprehensively search all exposed surface areas for damage or leaks can be distributed over multiple inspection agents to exploit their ability to move about freely and autonomously in confined spaces, and to perceive and communicate information. Combining many such miniature mobile robots into a collaborative multi-agent system can enable efficient and high-confidence inspection (ref. 1) thus replacing much of the anticipated human intervention required in the maintenance of future space transportation and habitation systems.

At NASA Glenn Research Center (GRC), research is underway to demonstrate the feasibility of a multi-robotic inspection system (ref. 2). This paper will provide an overview of the ongoing work involved in developing a smallscale functional robotics demonstration facility that will be used to validate the ability of autonomous robotic sensor platforms to perform cooperative inspection operations. Progress will be discussed in technology areas including: the hardware development of demonstration robotic platforms, the implementation of a hardware test-bed facility, the development of a three-dimensional software simulation environment, and the investigation of collaborative control algorithms. 


\section{Concept}

Interest in the study of multi-robot collaborative systems and their ability to complete complex tasks has grown in recent years (refs. 3 to 6) due in part to the increase in potential applications where it is necessary or useful to send multiple unmanned vehicles or robots into environments that are inaccessible or inhospitable to humans. The motivation for employing multi-agent systems is that they generally allow for robustness and redundancy unachievable through centralized, single-agent methods (refs. 7 and 8). Robustness is achieved since, as a group they can accept some attrition and reassign surviving resources to cover affected areas, rather than depend entirely on the reliability of a single, more complex robot. It is envisioned that an inspection team of robots could transport a variety of sensors over large areas faster, more extensively, and with less human involvement than Extra-Vehicular Activity (EVA) or tele-operation, and do so at lower cost.

\section{A. Motivation, Current Practice, and State-of-the-Art}

Although current inspection procedures for manned space systems are tedious, time-consuming and laborintensive, it is nonetheless a critical and indispensable process that ensures the safe operation of these systems. A key example is the need for thorough, high-confidence inspection for leaks. The Space Shuttle and International Space Station (ISS) have each suffered multiple leaks that have caused delays as well as safety concerns. In addition, potentially explosive fuel leaks in the Space Shuttle hydrogen fuel system led to the temporary grounding of the fleet in 1990 and have resulted in delays on several occasions. The leak detection system used on the Shuttle launch pad was a mass spectrometer connected to an array of sampling tubes placed throughout the network of fuelcarrying pipes. This system has several drawbacks which include transport delays between the fuel pipes to the sampling tubes, and insulation on the pipes masking the precise leak source since the hydrogen can travel for some distance inside the insulation (ref. 9).

Air leaks aboard the ISS are particularly serious because it is essentially a self-contained environment that must support long-term human habitation. Even a leak that reduces air pressure slowly can cause some equipment to fail. As an example, aboard the ISS is an air contaminant monitor that is certified for use only above $13.9 \mathrm{lb} / \mathrm{in}^{2}{ }^{2}$ (ref. 10). A troublesome air leak in the ISS in 2004 took two weeks to locate once it was discovered. (ref. 11). In order to find the leak, the astronauts "listened" with an ultrasonic detector, which picks up and amplifies the "rushing" sound of the gas escaping through an orifice (ref. 12). If this had not worked, some of the next search steps would have involved closing hatches between the U.S. and Russian segments, or even individual modules, in an attempt to rule out various locations, thereby narrowing down the potential leak sites (ref. 13).

Currently, months-long missions aboard the ISS are the longest humans endure, and emergency access to supplies or even to the earth are possible through the U.S. or Russian space shuttles. As space missions become longer term and reach farther out into space, the requirement for more robust, faster and more reliable leak detection systems becomes critical. Without earth proximity, vehicles and planetary bases will need to be completely selfsufficient. Thus the development of new space-based approaches for inspection and repair systems that are less labor-intensive is a prerequisite for future exploration missions. In fact, advancements in leak-detecting inspection and repair systems for space-based application are being reported (refs. 14 and 15).

This new requirement combined with emerging sensor technology (ref. 15) suggests the viability of the mobile sensor platforms concept for inspection and potentially repair. Furthermore, the idea of using mobile sensor platforms to perform inspection has precedent in terrestrial applications. Since the 1960s, the oil pipeline industry has used "smart pigs" to move through the oil pipes, carrying a variety of sensors to look for corrosion, coating disbondment, cracks, gouges, dents, buckles, wrinkles, and bending strain (ref. 16), as an early warning system, allowing repair before a leak can occur.

\section{B. Approach}

As a preliminary step towards realizing the long-term goal of an operational multi-robotic system capable of high-confidence inspection, the current work focuses on the design and development of a demonstration test-bed environment that features a team of autonomous, mobile demonstration robots that will allow the integration of control algorithms with actual hardware. This demonstration test-bed will allow researchers to develop algorithms and carry out experiments in an effort to validate the feasibility of using multi-agent robotics to perform cooperative, efficient, and effective inspections in applications such as the detection of leaks in future space transportation and habitation systems. 


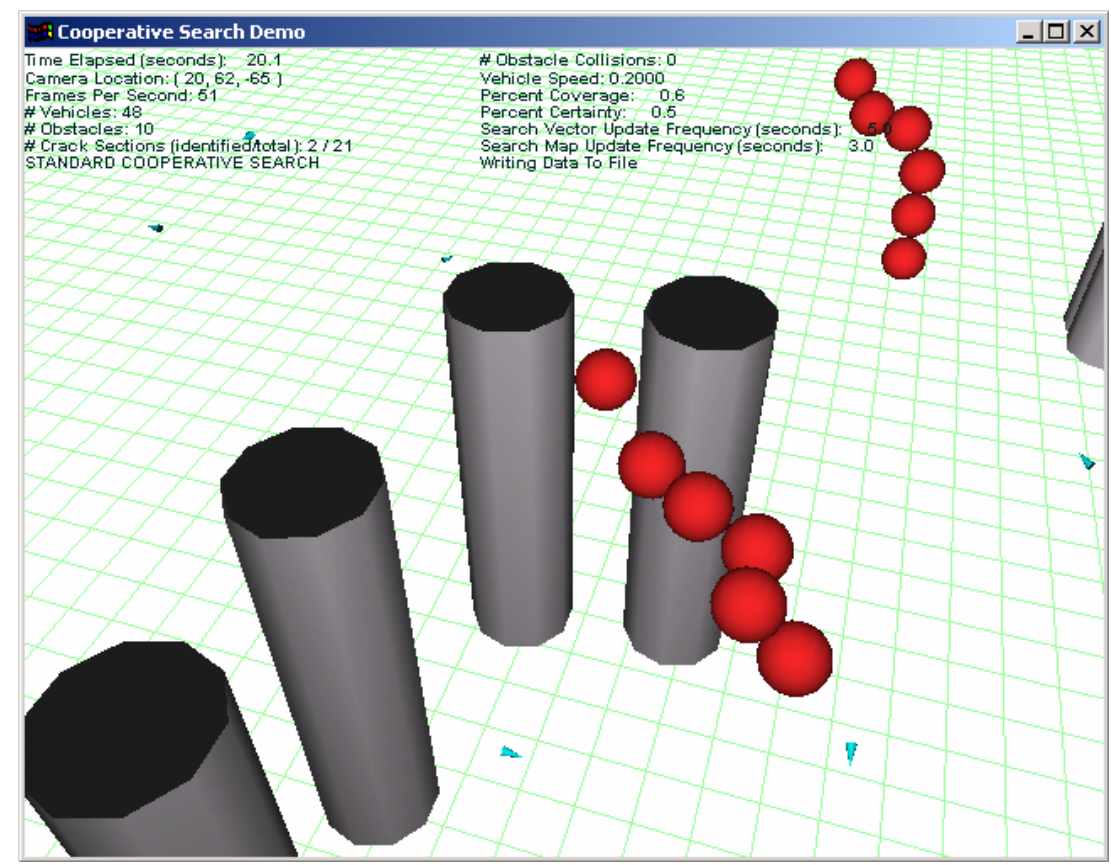

Figure 1.-Graphical simulation environment.

\section{Software Simulation Environment}

An interactive three-dimensional graphical simulation test-bed environment has been created to facilitate the development of multi-robot cooperative control algorithms. This environment serves as an important tool to assist in the design of algorithms prior to testing and validation on the hardware demonstration test-bed.

This virtual graphical test-bed was coded in C++ using the OpenGL graphics library and allows for smooth realtime three-dimensional motion graphics as well as highly interactive manipulation of the observation viewpoint. The software allows the modeling of three-dimensional environments replete with obstacles and barriers. To maintain generality, objects in the environment are modeled as simple three-dimensional forms: robotic agents are modeled as cones, targets as spheres, and obstacles as cylinders (fig. 1). These forms can be utilized as building-blocks for more complex forms. Agents within this environment are mathematically modeled as simple point-masses that travel at a constant velocity with full six degrees-of-freedom steering. Control of the movement and cooperative actions of the agents are provided by "plug-in" control logic modules. This plug-in architecture allows researchers to quickly implement and test various cooperative search and control algorithms, and allows them to demonstrate the resulting multi-agent behavior graphically.

\section{Hardware Demonstration Test-Bed}

A robotics demonstration test-bed has been constructed at NASA GRC to provide a facility that will allow robotics researchers to implement and test their multi-robot algorithms on a real hardware system and enable them to validate the feasibility of using autonomous robotic sensor platforms to perform inspection operations. The demonstration test-bed resides in a laboratory environment and consists of an 8 by $6 \mathrm{ft}$ horizontal arena populated by a team of demonstration robots that serves as the hardware platform upon which control algorithms can be implemented and tested (fig. 2). The arena design is flexible, with movable obstacles of arbitrary shape and quantity. Electric current-carrying copper tape is used to make the obstacles and arena boundaries detectable by the robots' proximity sensors.

The arena is surrounded by an optical motion-capture system manufactured by PhaseSpace Inc. This system will be used to track the positions of each robot in real-time during experiments. The motion-capture data can be used as a control input for online processing/analysis while the experiment is running, or kept as a record of the experiment for off-line analysis. 


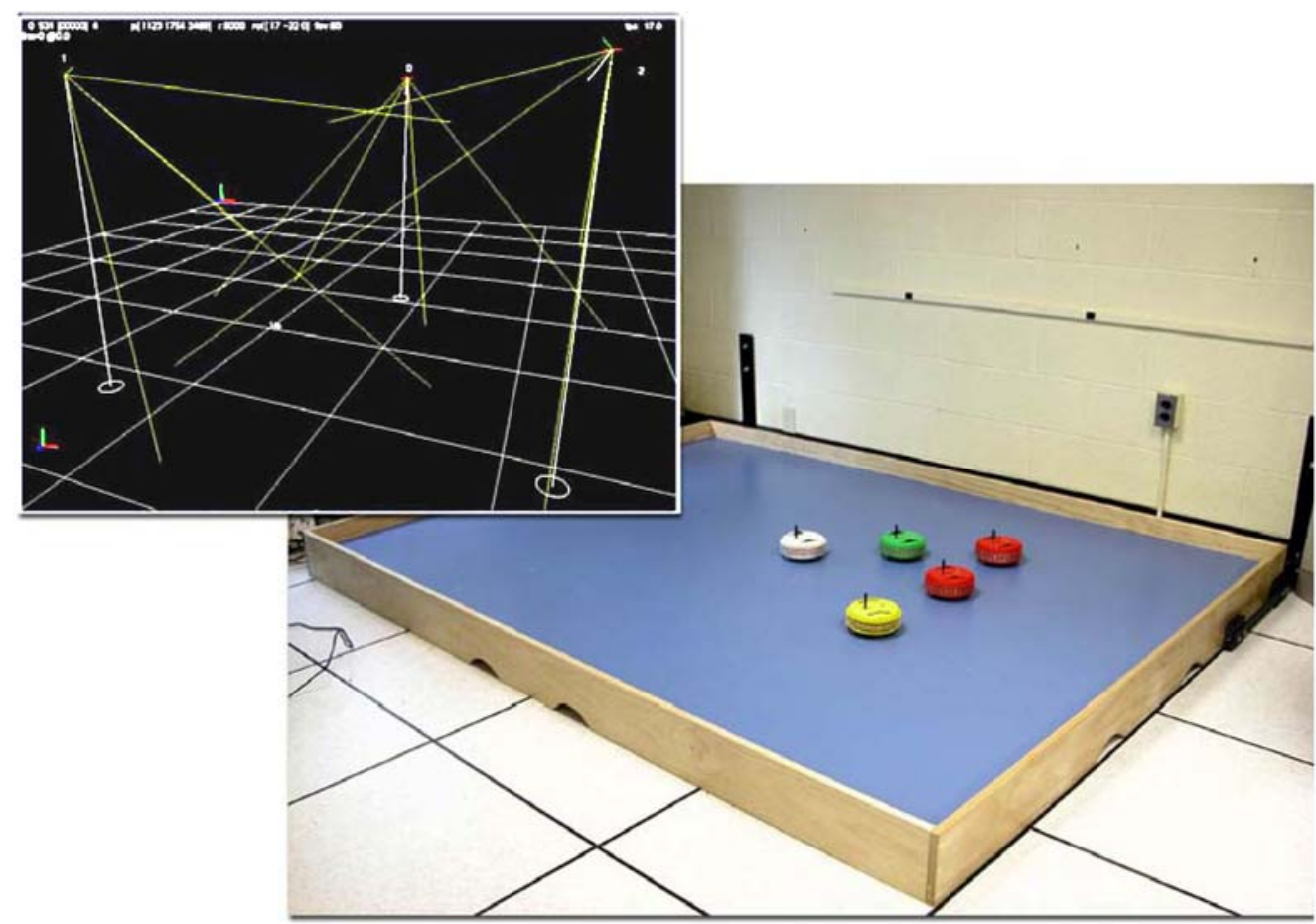

Figure 2.--Robotics testbed arena and phasespace screen view.

This measurement system is composed of a Linux-based server/hub connected to linear Charge Coupled Device (CCD) cameras, each of which possesses a $60^{\circ}$ conic field of view. Three such cameras are ceiling mounted and have been positioned and calibrated to allow full view of the entire arena surface. The CCD cameras are used to track Light-Emitting Diode (LED) markers mounted on each of the robots. Each of these LED markers is set with a unique flashing frequency by a wireless control box. As a result, the tracking system is capable of differentiating the various markers. The system perceives markers at a maximum of 480 frames per second. The position of each marker, in terms of Cartesian coordinates, can be reported with a latency of $20 \mu$ sec over an Ethernet connection, thereby allowing the tracking system to track and record the position and orientation of each robot over time during the course of experimental runs.

Although restricted to two-dimensional motion, the demonstration test-bed will allow for the integration and validation of cooperative search algorithms with real hardware robots. This capability is invaluable since it provides the opportunity to confront the challenges and limitations associated with implementation on a real-world system. Additionally, it will enable the transition of the concepts to a more realistic three-dimensional test-bed.

\section{Robot Development}

Key to the functionality of the test-bed is a group of demonstration robots that were developed using a novel puck-like design. These Puckbots are the latest generation in a line of multi-purpose demonstration robotic platforms that have been developed at NASA GRC over the past eight years (ref. 17). The heart of the robot is a custom designed circuit board (fig. 3) that supports duel 8051-type microcontrollers (manufactured by Silicon Laboratories Inc.) to supply the robot's processing capability. Locomotion is provided by two independent wheels driven by separate motors and power is provided by an onboard Lithium-ion battery.

Each Puckbot has two primary sensing capabilities. The first is a proximity sensing capability provided by a capacitance ring developed at GRC that takes advantage of the effects of capacitance to detect and determine the relative orientation of obstacles and other robots. This capacitance ring is comprised of a thin film of electrically conductive copper tape mounted along the perimeter of the robot and serves functionally as one plate of a capacitor. A similar capacitive surface on other robots, obstacles and boundaries serves as the other capacitor plate. As a robot approaches another object, the capacitance varies inversely with the distance between capacitor surfaces, thus enabling object and environment detection. An additional function of the capacitance ring is that by modulation of the capacitance field, it is possible to encode messages and allow for short-range communication between nearby robots. 


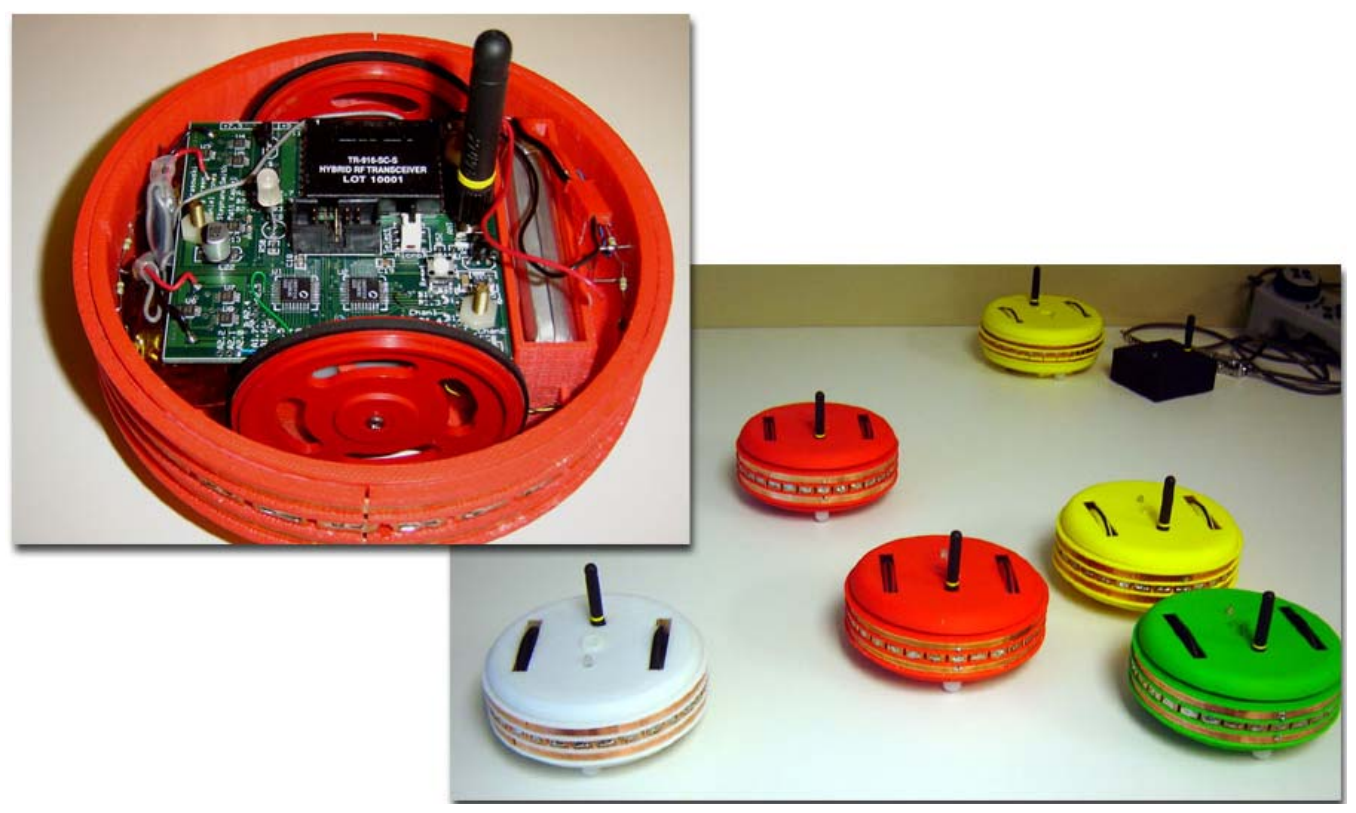

Figure 3.-Demonstration robots.

The second sensing capability is provided by an optical mouse sensor (manufactured by Agilent Technologies) mounted on the bottom of the robot and is used to detect displacement as the robot moves, thus providing navigation capability in the form of odometry via dead-reckoning. This sensor can also be used for visual detection of surface features and will allow the robots to locate targeted features on the arena surface.

Each robot has the capability to communicate with other robots as well as with a central computer through a twoway radio-frequency transceiver (manufactured by Linx Technologies). Several different types of communication are possible, including peer-to-peer (robot-to-robot or robot-to-master) and broadcasting (all listeners hear and process the message). A Media Access Control (MAC) protocol based on Karn’s Multiple-Access Collision Avoidance (MACA) (ref. 18) was developed at GRC and implemented on these radio-frequency transceivers. The protocol was designed to permit a wireless network of as many as ten mobile robots to communicate efficiently, effectively, and autonomously.

Networking, wireless networking in particular, is a complicated issue. Wireless networks suffer from several well-known problems. The first is "carrier sense" which is the ability for a particular node to know if the channel is busy. Problems are caused by nodes coming into and out of range of other nodes at arbitrary times and resulting in collisions where both data packets are lost (ref. 19). There are usually many different layers to a networking protocol, such as the 802.11 Standard for wireless networks introduced by IEEE in 1990. The 802.11 Standard makes specifications for two layers, the physical layer and the MAC layer (ref. 20). The physical layer is dictated by the hardware, and the MAC layer is specified in software. The MAC layer creates a set of rules for when and how nodes should transmit, with the goals of maximum channel utilization and minimum data loss. The MAC layer is highly important in all networked systems, and therefore must be carefully implemented in a multi-robot system to ensure robustness and efficiency. Karn's MACA was chosen as the basis for the GRC Puckbot's MAC protocol because of its ability to deal with collisions without needing carrier-sensing ability. Carrier sensing is often an approximate science, and a system that avoids the need for it would be more flexible and robust. Also, MACA is straight-forward, simple, and easy to implement. These are important qualities in multi-robot systems because each robot often has limited memory and computing resources. The GRC Puckbots, for instance, are limited to $16 \mathrm{kB}$ of program memory per microcontroller. A complex communication scheme could easily consume a large portion of this available memory. As currently implemented, the communication scheme will permit inter-robot, robot-to-host, and broadcast messages thus providing the communication backbone that will make it possible to implement higherlevel cooperative inspection algorithms that will be investigated.

\section{Research Issues}

Mobile robotics research is inherently multidisciplinary drawing on such areas as control theory, information theory, and artificial/computational intelligence. Some of the most prominent laboratories, centers and initiatives 
dealing with mobile robotics research include CMU's Robotics Institute (ref. 21), Georgia Tech’s Center for Robotics and Intelligent Machines (ref. 22), the (former) Developmental Robotics Laboratory on the Savannah campus of Georgia Tech (ref. 23), Stanford's AI Lab (ref. 24), MIT's CSAIL (ref. 25), USC (ref. 26), NASA's Jet Propulsion Lab (JPL) (ref. 27), JARA (Japan) (ref. 28), and Euron (Europe) (ref. 29). Some of the latest achievements of research into mobile robotics include NASA's Mars rovers, Spirit and Opportunity (ref. 30), and Stanford's Stanley, the winner of DARPA's Grand Challenge (ref. 31). The latest research findings are typically published in journals, such as IEEE Transactions on Autonomous Robotics (ref. 32), Elsevier's Robotics and Autonomous Systems Journal (ref. 33), and SAGE's International Journal of Robotics Research (IJRR) (ref. 34), conference proceedings, such as ICRA (ref. 35), IROS (ref. 36), and the recent (2005-06) RSS conferences (ref. 37), and books recently published by MIT Press (ref. 38).

A literature survey was conducted to identify recent (from the last few years) advances in mobile robotics research that are directly applicable to the GRC test-bed, and to the autonomous inspection scenarios of interest to NASA. The guiding principle for this investigation was to identify common fundamental open research issues for the autonomous operation of a group of mobile robots, which are enabling for the inspection application. As such, the following areas have been identified.

\section{A. Mapping}

Mobile robots can be deployed to explore their surroundings or the environment in which they need to operate. A mobile robot can be endowed with the capability to acquire a representation of its environment, or a map, based on its sensor measurements of specific features, such as objects and landmarks. Once acquired, the map can then be used to guide future actions. Research issues dealing with mapping include the ability to identify landmarks (ref. 39) and maintaining a map in a dynamic environment (ref. 40). Therefore, mapping its surroundings is one of the most important tasks that a mobile robot can perform, but that cannot be carried out without the robot being able to localize itself, as described next.

\section{B. Localization}

A mobile robot must first be able to localize itself, that is, to be able to assert its own location relative to its environment, in order to be able to function or carry out any meaningful task. For autonomous operation, a mobile robot must be able to localize itself using its onboard sensors. Such sensors can include a Global Positioning System (GPS) receiver in the case of terrestrial outdoor robots. However, GPS cannot be used for indoor robots or for robots with limited size or processing capabilities. In that case, the mobile robot must be able to use its onboard sensors to estimate where it is located in the environment based on physical measurements of its surrounding. Doing so involves dealing with the inherent uncertainty associated with estimating where the robot is at any given point in time. Several control theoretic approaches have been proposed to solve the robot location estimation problem. They include the Kalman filter (ref. 41) and its extended version, particle filters, and most recently, the Rao-

Blackwellized particle filter (RBPF) (ref. 42). Results of a recent approach for multi-robot localization are presented in reference 43.

\section{Simultaneous Mapping and Localization (SLAM)}

An interesting conundrum arises from the mapping and localization aspects of operating a mobile robot: a mobile robot can localize itself if it has a map, but it needs to be able to localize itself in order for it to build a map of its surroundings in the first place. Solving this problem gave rise to a class of algorithms to handle both of them simultaneously, namely: Simultaneous Localization and Mapping (SLAM) algorithms.

SLAM algorithms were developed over the past decade to enable a robot to incrementally build a map of its surroundings, based on measurements obtained from onboard sensors and, at the same time, determine its own position within the environment. A recent tutorial on SLAM appeared in reference 44. Most SLAM algorithms proposed to date are intended for the operation of a single mobile robot, whether indoor or outdoor. Recently published results include CSLAM (ref. 45), FastSLAM (refs. 46 and 47), Grid-based SLAM (2005) (ref. 48), GraphSLAM (ref. 49), and RBPF for SLAM (ref. 50).

\section{Algorithms for Multi-Robot SLAM}

SLAM algorithms are of limited value in the current research setting and test-bed at NASA GRC (described in section IV) for multi-robot operation. What is needed is a multi-Robot SLAM algorithm that could be used to deploy the robots for the target inspection task. Surprisingly, little work seems to be currently ongoing in this area. This can be due to the current priorities of funding agencies, such as DARPA's focus on autonomous navigation of a single mobile robot equipped with an array of sophisticated sensors (e.g., LADAR), or to the lack of multi-robot test-beds (since they are typically expensive and resource-intensive to acquire and maintain). Among published results, only three references were found: reference 51 details an experimentally validated particle filter-based SLAM for multi- 
robots, reference 52 presents a decentralized scheme for multi-robot localization, and reference 53 describes a probabilistic approach for cooperative localization and mapping.

\section{E. Higher Level Algorithms}

Deploying multiple robots to perform an inspection task collectively requires developing decentralized higher level algorithms for autonomous decision-making. Algorithms in the following categories have been identified for further investigation and experimentation using the GRC robotics demonstration test-bed.

1. Autonomous Decision-Making

Promising approaches for autonomous decision-making include the DAC5 architecture (ref. 54), a Bayesian decision-theoretic approach (ref. 55), and a new information gain-based approach described in reference 56. In the inspection and repair applications, all decisions that a robot needs to make, namely decisions involving locomotion, sensing and communication actions, should be treated in a uniform manner, thereby providing a unifying decision-making framework for the autonomous robot.

2. Collaborative Behavior

Common challenges for multi-robot operation can be found in three areas. The first area relates to processes that enable team formation, such as formation stabilization (ref. 57), self-organization (ref. 58) and routing (e.g., using an auction-based technique) (ref. 59). The next area relates to the concepts of cooperative localization (ref. 45) and cooperative SLAM (ref. 52). The final area of interest relates to the ability to perform multi-robot localization (ref. 59).

3. Swarm

A fundamentally different way of deploying multiple robots is the swarm approach (ref. 60). Relevant results from this area include avoidance behavior (ref. 61), coverage (ref. 62), task assignment (ref. 63), and flocking (ref. 64).

4. Pattern Recognition

A mobile robot must perform some sort of fusion of sensor information to draw conclusions about its environment from its potentially varied sensing capabilities. Dealing with sensor data also requires performing pattern recognition, which in turn involves algorithms for feature selection, extraction and classification of sensor data. Recent advances in these areas can be found in the proceedings of the 11th Online World Conference on Soft Computing in Industrial Applications (WSC11) (ref. 65).

\section{Conclusion}

A functional robotics demonstration facility has been constructed at NASA GRC that will enable researchers to experiment and test the feasibility of future multi-robotic inspection systems. This paper provided an overview of the project and presented the status of the various components. An interactive three-dimensional graphical simulation test-bed environment has been developed and is currently being used to facilitate the design and testing of high level cooperative algorithms. Demonstration robots have been constructed and a robust wireless network communication protocol has been developed to enable crucial communication capability. A robotic test-bed has been completed and will allow for real-world experimentation to validate cooperative control algorithms. In addition, a thorough literature survey was conducted to identify recent advances in mobile robotics research that are directly applicable to the GRC test-bed. This survey will provide guidance for the ongoing investigation and test-bed experimentation efforts that will help demonstrate the feasibility of an autonomous multi-robotic inspection system.

With the test-bed fully functional, researchers will now proceed to develop high-level controllers for robotic action and behavior (that build upon the existing lower level code for locomotion, sensing and communication), and develop experiments for online and offline multi-robot SLAM algorithms. Subsequently, high level collaborative algorithms will be implemented and examined while measuring performance using several techniques under various operational conditions for the application scenarios of interest, namely, inspection and repair.

\section{References}

1. Gary W. Hunter, "Morphing, Self-Repairing Engines: A Vision for the Intelligent Engine of the Future,” AIAA/ICAS International Air \& Space Symposium, AIAA-2003-3045, July 14-17, 2003.

2. Litt, J., Wong, E., Krasowski, M., Greer, L. Cooperative Multi-Agent Mobile Sensor Platforms for Jet Engine Inspection-Concept and Implementation. KIMAS, Boston, 2003. pp. 716-721.

3. Cao, Y.U., Fukunaga, A., and Kahng, A. "Cooperative mobile robotics: Antecedents and directions."

Autonomous Robots, 4:1-23. 1997. 
4. Gage, D. "How to Communicate with Zillions of Robots." Proc. of SPIE Mobile Robots VIII, Boston, 9-10 September 1993, Volume 2058, pp. 250-257.

5. Wong, E., Litt, J. “Autonomous Multi-Agent Robotics for Inspection and Repair of Propulsion Systems.” AIAA 1st Intelligent Systems Technical Conference, 2004.

6. Gerkey, B., Mataric, M. “Principled Communication for Dynamic Multi-Robot Task Allocation.” Experimental Robotics VII, LNCIS 271, 2001, pp. 353-362.

7. Gage, D. "How to Communicate with Zillions of Robots." Proc. of SPIE Mobile Robots VIII, Boston, 9-10 September 1993, Volume 2058, pp. 250-257.

8. Liu, Y., Passino, K. “Swarm Intelligence: Literature Overview.” Ohio State University. March 2000.

9. Hunter, G.W., Xu, J.C., Liu, C. -C., Makel, D.B., "Microfabricated Chemical Sensors for Aerospace Applications," in MEMS: Design and Fabrication, Gad-el-Hak, Mohamed (ed), CRC Press, Taylor \& Francis Group, Boca Raton, FL, 2006.

10. Dunn, Marcia, "Search for Leak on Space Station Widens," posted: 03:00 pm ET, 09 January 2004, http://www.space.com/missionlaunches/exp8_update_040109.html, accessed: August 11, 2006.

11. Banke, Jim, “Convenient Crew Handhold Caused Station Air Leak,” posted: 07:30 pm ET, 23 January 2004, http://www.space.com/missionlaunches/exp8_update_040123.html, accessed: August 11, 2006.

12. “Aircraft Leak Detection,” http://www.gesensing.com/products/resources/datasheets/age1401.pdf, accessed: August 11, 2006.

13. Banke, Jim, “Station Crew Hunts for Elusive Air Leak,” posted: 07:30 pm ET, 06 January 2004, http://www.space.com/missionlaunches/exp8_update_040106.html, accessed: August 11, 2006.

14. Fronczek, J.W., and Prasad, N.D., "Bio-Inspired Sensor Swarms to Detect Leaks in Pressurized Systems," IEEE International Conference on Systems, Man, and Cybernetics, October 10-12, 2005.

15. Hunter, G.W., Xu, J.C., Neudeck, P.G., Makel, D.B., Ward, B., and Liu, C. -C., "Intelligent Chemical Sensor Systems for In-Space Safety Applications,” AIAA-2006-4356, 42nd Joint Propulsion Conference, Sacramento, CA, July 9-12, 2006.

16. Construction Engineering \& Management, Purdue University, "Emerging Construction Technologies—Smart Pigs,” http://www.new-technologies.org/ECT/Mechanical/smartpigs.htm, accessed: August 14, 2006.

17. Michael J. Krasowski, Lawrence C. Greer, and Lawrence G. Oberle, "Mobile Sensor Technologies Being Developed," Research \& Technology 2002, NASA/TM-2003-211990.

18. Karn, P. "MACA—A New Channel Access Method for Packet Radio.” Proc. CNC, London, Ontario, Canada, Sep. 1990, vol. 1, pp. 134-140.

19. Haas, Z., Tabrizi, S. “On Some Challenges and Design Choices in Ad-Hoc Communications.” IEEE MILCOM 1998.

20. "Introduction to the IEEE 802.11 Wireless LAN Standard.” Raylink and Raytheon Electronics. June 2001

21. http://www.ri.cmu.edu/.

22. http://www.robotics.gatech.edu/.

23. http://www.gtsav.gatech.edu/drl.

24. http://ai.stanford.edu/.

25. http://www.csail.mit.edu/index.php.

26. http://robotics.usc.edu/.

27. http://claraty.jpl.nasa.gov/main/overview/publications/index.html.

28. http://www.jara.jp/e/.

29. http://www.euron.org/.

30. http://marsrovers.nasa.gov/home/.

31. http://www.darpa.mil/grandchallenge/index.asp.

32. http://www.ieee.org/portal/pages/pubs/transactions/tra.html.

33. http://www.elsevier.com/locate/robot.

34. http://ijr.sagepub.com/.

35. http://www.ncsu.edu/IEEE-RAS/RAS/RASconference.html.

36. http://www.iros2004.org/.

37. http://www.roboticsproceedings.org/rss01/.

38. http://mitpress.mit.edu/.

39. Ranganathan, A., Dellaert, F. “A Rao-Blackwellized Particle Filter for Topological Mapping,” ICRA 2006, Orlando, FL, http://www-static.cc.gatech.edu/ ananth/pub/icra06.pdf.

40. Biber, P.,Duckett, T. "Dynamic Maps for Long-Term Operation of Mobile Service Robots," Proceedings of Robotics: Science and Systems, June 2005, Cambridge, USA, 
http://www.roboticsproceedings.org/rss01/p03.html, Biber-RSS-05.

41. Abbeel, P., Coates, A., Montemerlo, M., Ng, A., Thrun, S. "Discriminative Training of Kalman Filters," Abbeel-RSS-05, Proceedings of Robotics: Science and Systems, June 2005, Cambridge, USA, http://www.roboticsproceedings.org/rss01/p38.pdf.

42. Kwok, C., Fox, D., Meila, M. “Real-time Particle Filters,” Proceedings of the IEEE, 92(2), 2004, http://www.cs.washington.edu/ai/Mobile_Robotics/.

43. Roumeliotis, S., Bekey, G. “Distributed Multi-Robot Localization,” IEEE Transactions on Robotics and Automation, 18(5), pp. 781-795, Oct. 2002, http://www-users.cs.umn.edu/ stergios/publications.html.

44. Durrant-Whyte, H., Bailey, T. "Simultaneous localization and mapping: Part I,” IEEE Robotics and Automation Magazine, Volume 13, Issue 2, June 2006, pp. 99-108.

45. Mourikis, A., Roumeliotis, S. "Performance Bounds for Cooperative Simultaneous Localization and Mapping (C-SLAM),” Proceedings of Robotics: Science and Systems, June 2005, Cambridge, USA, Mourikis-RSS-05, http://www.roboticsproceedings.org/rss01/p10.html.

46. M. Montemerlo, S. Thrun, D. Koller, and B. Wegbreit. FastSLAM: A factored solution to the simultaneous localization and mapping problem. In Proceedings of the AAAI National Conference on Artificial Intelligence, Edmonton, Canada, 2002.

47. Stachniss, C., Haehnel, D., Burgard, W. “Exploration with Active Loop-Closing for FastSLAM,” Proceedings of the IEEE/RSJ International Conference on Intelligent Robots and Systems (IROS), pp. 1505-1510, Sendai, Japan, 2004.

48. Grisetti, G., Stachniss, C., Burgard. W. "Improving Grid-based SLAM with Rao-Blackwellized Particle Filters by Adaptive Proposals and Selective Resampling,” Proceedings of the IEEE International Conference on Robotics and Automation (ICRA), pp. 2443-2448, Barcelona, Spain, 2005.

49. Thrun, S., Montemerlo, M. "The GraphSLAM Algorithm with Applications to Large-Scale Mapping of Urban Structures,” Volume 25 Issue 5/6, May/June 2006, Special Issue on the Ninth International Symposium on Experimental Robotics, 2004 International Journal of Robotics Research (IJRR), SAGE Publications, http://www.ijrr.org/contents/25_05/IJR25_05.html.

50. RBPF for SLAM (2006).

51. Howard, A. "Multi-robot Simultaneous Localization and Mapping using Particle Filters," Proceedings of Robotics: Science and Systems, June 2005, Cambridge, USA, http://www.roboticsproceedings.org/rss01/p27.html, Howard-RSS-05.

52. Rekleitis, I., Dudek, G., Milios, E. "Multi-Robot Cooperative Localization: A Study of Trade-offs Between Efficiency and Accuracy,” IEEE/RSJ International Conference on Intelligent Robots and Systems, Lausanne, Switzerland, Oct. 2002, pp. 2690-2695.

53. Roumeliotis, S., Rekleitis, I. "Propagation of Uncertainty in Cooperative Multirobot Localization: Analysis and Experimental Results,” Autonomous Robots, 17(1), pp. 41-54, July 2004.

54. Howard, A., Mataric, M., Sukhatme, G. "Cooperative Relative Localization for Mobile Robot Teams: An Egocentric Approach,” Proceedings of the Naval Research Laboratory Workshop on Multi-Robot Systems, Washington, DC, March 17-19, 2003, pp. 65-76.

55. Verschure, P., Althaus, P. “A real-world rational agent: Unifying old and new AI,” Cognitive Science, 27(4):561-590, 2003.

56. Stachniss, C., Grisetti, G., Burgard, W. "Information Gain-based Exploration Using Rao-Blackwellized Particle Filters,” in Proceedings of Robotics: Science and Systems (RSS), pp. 65-72, Cambridge, MA, USA, 2005.

57. Tanner, H., Kumar, A. "Formation Stabilization of Multiple Agents Using Decentralized Navigation Functions," in Proceedings of Robotics: Science and Systems (RSS), pp. 65-72, Cambridge, MA, USA, 2005, Tanner-RSS-05, http://www.roboticsproceedings.org/rss01/p07.pdf.

58. Floreano, D., Urzelai, J., "Evolutionary Robots with on-line self-organization and behavioral fitness,” Neural Networks, 13(4-5), 2000, pp. 431-443.

59. Roumeliotis, S., Berkey, G. “Distributed Multirobot Localization,” IEEE Transactions on Robotics \& Automation, Volume 18, Number 5, October 2002.

60. http://www.swarm-bots.org/.

61. http://www.swarm-bots.org/hole-avoidance.html.

62. Silva, V., Ghrist, R., Muhammad, A. "Blind Swarms for Coverage in 2-D,” Proceedings of Robotics: Science and Systems, June 2005, Cambridge, USA, http://www.roboticsproceedings.org/rss01/p44.pdf, Silva-RSS-05.

63. McLurkin, J., Yamins, D. "Dynamic Task Assignment in Robot Swarms," Proceedings of Robotics: Science and Systems, June 2005, Cambridge, USA, http://www.roboticsproceedings.org/rss01/p18.html, McLurkinRSS-05. 
64. Hayes, A., Dormiani-Tabatabaei, P. "Self-Organized Flocking with Agent Failure: Off-Line Optimization and Demonstration with Real Robots,” Proceedings of the 2002 IEEE International Conference on Robotics and Automation IROS-02, May 2002, Washington DC, USA, pp. 3900-3905, http://swis.epfl.ch/Backup_Caltech/CORO/publications.htm.

65. 11th Online World Conference on Soft Computing in Industrial Applications (WSC11), http://www.cs.armstrong.edu/wsc11/. 


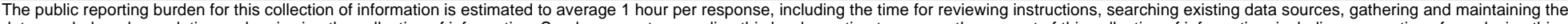

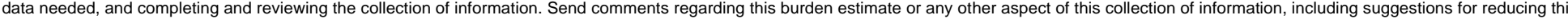

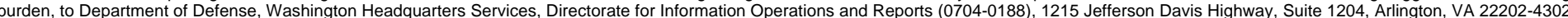

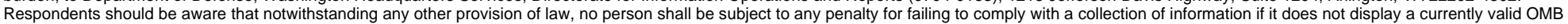
control number.

PLEASE DO NOT RETURN YOUR FORM TO THE ABOVE ADDRESS.

\section{REPORT DATE (DD-MM- $Y Y Y Y)$ \\ 2. REPORT TYPE \\ 3. DATES COVERED (From - To)}

25-06-2007

\section{TITLE AND SUBTITLE}

Towards Autonomous Inspection of Space Systems Using Mobile Robotic

Sensor Platforms

\section{5a. CONTRACT NUMBER}

5b. GRANT NUMBER

5c. PROGRAM ELEMENT NUMBER

5d. PROJECT NUMBER

Wong, Edmond; Saad, Ashraf; Litt, Jonathan, S. 5f. WORK UNIT NUMBER

WBS 457280.02.07.03.02

\section{PERFORMING ORGANIZATION NAME(S) AND ADDRESS(ES)}

National Aeronautics and Space Administration

John H. Glenn Research Center at Lewis Field

Cleveland, Ohio 44135-3191

\section{PERFORMING ORGANIZATION \\ REPORT NUMBER}

E-16052

\section{SPONSORING/MONITORING AGENCY NAME(S) AND ADDRESS(ES)}

National Aeronautics and Space Administration

Washington, DC 20546-0001

and

U.S. Army Research Laboratory

Adelphi, Maryland 20783-1145

\section{DISTRIBUTIONIAVAILABILITY STATEMENT}

Unclassified-Unlimited

Subject Category: 63

Available electronically at http://gltrs.grc.nasa.gov

This publication is available from the NASA Center for AeroSpace Information, 301-621-0390

\section{SPONSORING/MONITORS ACRONYM(S) \\ NASA, ARL}

11. SPONSORING/MONITORING REPORT NUMBER

NASA/TM-2007-214838; ARL-TR-3930;

AIAA-2006-7411

\section{SUPPLEMENTARY NOTES}

\section{ABSTRACT}

The space transportation systems required to support NASA's Exploration Initiative will demand a high degree of reliability to ensure mission success. This reliability can be realized through autonomous fault/damage detection and repair capabilities. It is crucial that such capabilities are incorporated into these systems since it will be impractical to rely upon Extra-Vehicular Activity (EVA), visual inspection or tele-operation due to the costly, labor-intensive and time-consuming nature of these methods. One approach to achieving this capability is through the use of an autonomous inspection system comprised of miniature mobile sensor platforms that will cooperatively perform highconfidence inspection of space vehicles and habitats. This paper will discuss the efforts to develop a small scale demonstration test-bed to investigate the feasibility of using autonomous mobile sensor platforms to perform inspection operations. Progress will be discussed in technology areas including: the hardware implementation and demonstration of robotic sensor platforms, the implementation of a hardware test-bed facility, and the investigation of collaborative control algorithms.

\section{SUBJECT TERMS}

Multi-agent systems; Robotics; Robot; Automated inspection; Sensors; Autonomy; Swarm control

\begin{tabular}{|c|c|c|c|c|}
\hline 16. SECURI & ASSIFICATION & & 17. LIMITATION OF & 18. NUMBER \\
\hline $\begin{array}{l}\text { a. REPORT } \\
\mathrm{U}\end{array}$ & $\begin{array}{l}\text { b. ABSTRACT } \\
U\end{array}$ & $\begin{array}{l}\text { c. THIS } \\
\text { PAGE } \\
\text { U }\end{array}$ & & $\begin{array}{c}\text { PAGES } \\
16\end{array}$ \\
\hline
\end{tabular}

\section{9a. NAME OF RESPONSIBLE PERSON} Jonathan S. Litt 19b. TELEPHONE NUMBER (include area code) 216-433-3748 

